\title{
Right Ventricular Failure Following Left Ventricular Assist Device Implantation
}

\author{
Niraghatam Harshavardhan ${ }^{1 *}$, Amitabh Satsangi ${ }^{2}$ \\ ${ }^{1}$ Department of CTVS, Sri Sathya Sai Institute of Higher Medical Sciences, Bengaluru, India \\ ${ }^{2}$ Department of Cardiothoracic and Vascular Surgery, All India Institute of Medical Sciences, New Delhi, India \\ Email: *vardhan87@gmail.com, amoeba418@gmail.com
}

How to cite this paper: Harshavardhan, N. and Satsangi, A. (2020) Right Ventricular Failure Following Left Ventricular Assist Device Implantation. World Journal of Cardiovascular Surgery, 10, 243-253. https://doi.org/10.4236/wjcs.2020.1012026

Received: October 27, 2020

Accepted: December 6, 2020

Published: December 9, 2020

Copyright $\odot 2020$ by author(s) and Scientific Research Publishing Inc. This work is licensed under the Creative Commons Attribution International License (CC BY 4.0).

http://creativecommons.org/licenses/by/4.0/

(c) (i) Open Access

\begin{abstract}
Left ventricular assist device (LVAD) is being used increasingly in recent years for end stage heart failure as a bridge to transplant (BTT) and also as a destination therapy (DT). Patients with end stage heart failure have some degree of elevated pulmonary capillary wedge pressure, causing right ventricular hypertrophy which in due course leads to decreased dilatation of the RV and fall in cardiac output \& severe tricuspid regurgitation (TR) presenting with features of RV failure (RVF). Implantation of LVAD improves left heart function at the cost of right ventricular output with an incidence of $25 \%$ $30 \%$. RVF may lead to impaired LVAD flow, difficulty in weaning from cardio-pulmonary bypass (CPB), decreased tissue perfusion and multi-organ failure. In this article we comprehended the pathophysiology leading to RVF post LVAD implantation and its preoperative predictors and the various treatment modalities for managing RVF post LVAD implantation.
\end{abstract}

\section{Keywords}

Left Ventricular Assist Device (LVAD), Right Ventricular Failure (RVF), Right Ventricular Assist Device (RVAD), Right Ventricular Stroke Work Index (RVSWI), Tricuspid Regurgitation (TR)

\section{Introduction}

Increasing incidence of heart failure and limited availability of donor heart for transplantation made mechanical assist devices an important alternative which substitutes the pumping action of the failing ventricle and provides circulatory support maintaining the blood to flow to various organ systems of the body. Left ventricular assist device (LVAD) is one such mechanical assist device which is being used increasingly in recent years for end stage heart failure those with Interagency 
Registry for Mechanically Assisted Circulatory Support (INTERMACS) profile levels 2, $3 \& 4$ as a Bridge To Transplant (BTT) for patients on transplant waiting list and also as a Destination Therapy (DT) in whom transplant is not an option [1]. It is also used in various short term indications such as in patients presenting with cardiogenic shock after an acute myocardial infarction or following a cardiac surgery, myocarditis and in patients with refractory ventricular arrythmias. Working principle of LVAD is that it draws oxygenated blood from the apex of left ventricle from where the blood passes through a mechanical pump and is delivered into the aorta in a pressurized manner. There has been an evolution in the device from initial pulsatile LVADs to the present generation of continuous flow LVADs which are more durable and smaller in size facilitating easy implantation. LVAD implantation improves left heart function at the cost of right ventricular output. Optimal filling of LV from a normally functioning right ventricle (RV) is the norm for optimal functioning of LVAD. Patients with end stage heart failure have some degree of elevated pulmonary capillary wedge pressure, causing right ventricular hypertrophy which in due course leads to decreased dilatation of the RV and fall in cardiac output \& severe tricuspid regurgitation (TR) presenting with features of RV failure (RVF). Incidence of RV failure is about $20 \%-35 \%$ with an early onset RVF seen in $13 \%$ and late onset in $7 \%$ [2] [3]. RVF may lead to impaired LVAD flow, difficulty in weaning from cardio-pulmonary bypass (CPB), decreased tissue perfusion and multi-organ failure, which are associated with increased morbidity and mortality [4]. RVF post LVAD implantation is associated with prolonged hospital and ICU stay [2].

\section{Pathophysiology \& Diagnosis of RVF}

Biventricular failure is the common end point in stage D heart failure. Elevated left ventricular end diastolic pressures induce high pulmonary vascular resistance with PAH and subsequent RV failure. Initiation of LVAD causes acute drop in LVEDP and PCWP to restore cardiac output and relieve congestion of lungs. Kukucka et al. confirmed these findings in a perioperative Trans Esophageal Echocardiography (TEE) study with improved RV end-diastolic geometry as a result of significant reduction in RV afterload [4]. The pathophysiology of $\mathrm{RV}$ failure is attributed to increased preload, decreased contractility, and increased afterload, each of which can occur alone or in combination in the perioperative period. Improved left-sided forward flow with the help of implanted LVAD increases RV preload and alters the Frank-Starling mechanics for the already decompensated RV. This acute changes in RV hemodynamics worsen tricuspid regurgitation (TR), cause leftward bowing of the interventricular septum, and ventriculo-arterial uncoupling [5]. Mechanical factors such as proper positioning of inflow canula, optimal speed of LVAD pump in order to avoid suction effect, affect septal interdependence after LVAD implant and can affect RV function. Study by Topilsky et al. showed that persistent inadequate unloading of LV with left sided shift of inter-atrial and inter-ventricular septum causes in- 
crease in RV afterload and was associated with worse postoperative 90-day outcomes post LVAD implantation [6]. Acute unloading of the left ventricle leads to a septal shift that alters RV shape and size, thereby affecting its contractility [7] [8]. The already existing RV impairment might be manifested with the increase in right-sided preload afforded by LVAD perfusion [7]. These aspects should be routinely considered at the time of LVAD and when deciding on device settings to keep the septal shift to a minimum. For the purpose of maintaining uniformity Interagency Registry for Mechanically Assisted Circulatory Support (INTERMACS) defined criteria for the diagnosis of RVF post LVAD implantation in 2012 (Table 1) [9].

\section{Predictors and Risk Factors of RVF}

In the study by Kormos RL et al. baseline CVP and CVP/PCWP ratio was significantly higher in patients with early RVF, especially for patients requiring RVADs, when compared to those without RVF [2]. Mean Right Ventricular Stroke Work Index (RVSWI) was significantly lower for the early RVF group. Patients who are on high inotropic support, pressor agents, Intra Aortic Balloon Pump (IABP) support, who has sustained a cardiac arrest previously are high risk candidates for postoperative RVF. Matthews JC et al. developed a scoring system on the basis of the above parameters assigning a value of 4 if the patient requires a pressor agent preoperatively, a value of 2 if the serum Aspartate Transaminase levels $>80 \mathrm{IU} / \mathrm{ml}$, a score of 2.5 if serum Bilirubin levels are $>2$ $\mathrm{mg} / \mathrm{dl}$, a value of 3 if serum creatinine levels are $>2.3 \mathrm{mg} / \mathrm{dl}$ or if the patient requires

Table 1. INTERMACS diagnostic and severity criteria of RVF [9].

\section{Diagnostic criteria}

1. Symptoms and signs of persistent right ventricular dysfunction

2. Central venous Pressure (CVP) $>18 \mathrm{mmHg}$ with Cardiac Index $<2.0 \mathrm{~L} / \mathrm{min} / \mathrm{m}^{2}$; In the absence of elevated left atrial/pulmonary capillary wedge pressure $>18 \mathrm{mmHg}$, tamponade, ventricular arrhythmias or pneumothorax

3. Requiring (RVAD) Right Ventricular Assist Device implantation

4. Requiring inhaled nitric oxide

5. Inotropic therapy for duration of more than one week at any time after LVAD implantation

\section{Severity scale}

Severe: Requiring RVAD implantation

Moderate: Usage of inotropes or IV or inhaled pulmonary vasodilators such as iNO or prostaglandin E

Mild: Atleast 2 of the 4 criteria:

1. CVP $>18 \mathrm{mmHg}$ or mean RA pressure $>18 \mathrm{mmHg}$

2. $\mathrm{CI}<2.3 \mathrm{~L} / \mathrm{min} / \mathrm{m}^{2}$

3. Ascites or evidence of moderate to worse peripheral oedema

4. Evidence of elevated CVP by echocardiogram (dilated inferior vena cava without collapse), and in physical exam (signs of increased jugular venous pressure)

Reproduced with permission from Argiriou, M., Kolokotron, S.M., Sakellaridis, T., Argiriou, O., Charitos, C., Zarogoulidis, P., Katsikogiannis, N., Kougioumtzi, I., Machairiotis, N., Tsiouda, T., Tsakiridis, K. and Zarogoulidis, K. (2014) Right Heart Failure Post Left Ventricular Assist Device Implantation. Journal of Thoracic Disease, 6, S52-S59. 
a Renal replacement Therapy with a cumulative score of $>16$ predicting a high risk of postoperative RVF [3]. Several other risk scoring systems have been developed which predict the right ventricular failure during the postoperative period using various clinical and investigational parameters with variable sensitivities and specificities such as Fitz Patrick risk score $>50$ with a sensitivity of $83 \%$ and specificity of $80 \%$ [10], Drakos scoring system in which they classified the patients into 4 risk categories with an incidence of RVF of $11 \%$ in low risk group, and an incidence of $83 \%$ in high risk category [11].

White blood cell count and blood urea nitrogen (BUN) were higher in RVF group and required RVAD implantation. Increased WBC count $>10.4 \times 10^{3}$ [Odds ratio $2.2(1.20-3.70) \mathrm{P}<0.05$ ] and lower hematocrit values $<31 \%$ [Odds ratio $2.3(1.30-3.90) \mathrm{P}<0.001$ ] were independent risk factors among those who required Right Ventricular Assist Device (RVAD) support compared to those who did not require RVAD support [2]. In a study by Ochiai et al. of 245 patients, the need for pre-LVAD circulatory support, small BSA, female sex, elevated total serum bilirubin \& aspartate transferase levels and cardiomyopathy due to non-ischemic causes were found to independently predict RVF after LVAD placement [12]. In a study by Pagani et al. higher incidence of RV failure after LVAD implantation was noted in patients initially supported with ECMO before LVAD implantation. The authors suggested that this increased incidence could be attributed to a lung injury exacerbated by ECMO or may reflect the overall increased severity of illness and initial degree of hemodynamic instability [13]. Argiriou M et al. stated the incidence of RVF is higher among patients in whom LVAD implantation is performed as DT when compared to BTT patients. They predicted a higher probability of RVF post LVAD implantation in patients with central venous pressure (CVP) $\geq 8 \mathrm{mmHg}$, PCWP $\geq 18 \mathrm{mmHg}$, CVP/PCWP ratio $\geq 0.66$, pulmonary vascular resistance $(\mathrm{PVR})>2$ wood units [9]. Farrar et al. reported that a higher proportion of patients receiving an isolated LVAD had coronary artery disease (54\%) [14]. Presence of severe tricuspid regurgitation in the preoperative period and patients undergoing redo LVAD implantation have a high risk of developing RVF in postoperative period [15].

\section{Evaluation of Right Ventricular Function}

Prior to implantation, history of patient pertaining to right heart failure interrogated. History and physical signs of right heart failure in the form of lower limb edema, ascites, pulsatile liver and icterus has to be elicited. Patients with underlying or masked right heart failure can worsen after LVAD implantation and necessitate a Biventricular Assist Device (BiVAD).

The laboratory studies should be performed to determine nutritional status, renal function and hepatic function. Nutritional deficiencies occur in patients with severe heart failure; however, RV failure may substantially contribute to nutritional deficiencies via hepatic dysfunction secondary to passive hepatic congestion or protein-losing enteropathy. 
Cardiac catheterization includes determination Pulmonary Capillary Wedge Pressure (PCWP), Right Atrial Pressure (RAP), Pulmonary Artery Pressure (PAP), RVSWI and Fick cardiac output. RAP > $20 \mathrm{mmHg}$ and high CVP/PCWP ratio are indicators of the severity of RV function impairment. Optimal medical management before LVAD implantation can decrease the RAP to a range of $\leq 15$ mmHg. This response suggests that RV function will be adequate to support LVAD implantation [16].

Echocardiographic evaluation of RV morphology and contractile function begins with accurate measurement of the ventricular dimensions. Measures of RV mechanical performance must take into account the presence and severity of tricuspid and pulmonary valve pathologies in addition to PAP (i.e. RV afterload). Systolic function is often measured solely as the RV ejection fraction, which is sensitive to RV afterload, preload, valvular dysfunction, and inotropic stimulation. Kato TS et al. [17] in their study on 111 patients developed left ventricular echocardiographic RVF score based on Receiver Operator Characteristic (ROC) curve analysis where each $2 \mathrm{D}$ echo parameter was categorized and each category was assigned a score, and a cumulative score was developed. Left Ventricular End Diastolic Diameter values grouped as $>78 \mathrm{~mm}, 79 \mathrm{~mm}$ to $70 \mathrm{~mm}$, and $<70 \mathrm{~mm}$, LV ejection fraction grouped as $<19 \%, 19 \%-33 \% \& 33 \%$; and left atrial diameter/LVEDD ratio $<0.63,0.63$ to $0.68,>0.68$; each of the above parameter was graded as 0 and 1 point and 2 points, respectively. A cumulative score of $\geq 3$ predicted RVF with a sensitivity of $88.6 \%$ and score $\geq 5$ with a specificity of $80.3 \%$. In conclusion, patients with relatively small LV size, preserved LV contraction, and dilated left atrium were at higher risk for RVF after LVAD surgery. Thus LV echocardiographic RVF score is a noninvasive method which predicts RVF post LVAD implantation. The RV stroke work index (RVSWI) is a measurement of RV function. A low RVSWI has been associated with increased risk for severe RV failure requiring RVAD implantation. Argiriou $\mathrm{M}$ et al. predicted RVF in post LVAD implantation patients with RVSWI $\leq 400 \mathrm{mmHG}$ $\mathrm{ml} / \mathrm{m}^{2}$ on $2 \mathrm{D}$ echo [9]. Aissaoui et al. calculated Fractional Area Change (FAC) \%, Basal RV end diastolic diameter ( $\mathrm{mm}$ ), RV Tissue Doppler Systolic Velocity and TAPSE in the Assessment of Right Ventricle before the Implantation of Left Ventricular Assist Device by Echocardiographic Parameters (ARVADE) study, and suggested that an ARVADE score > 2 predicts RVF with $92 \%$ sensibility and $67 \%$ specificity [18]. Echocardiographic RV assessment by Grant et al. in their series of 117 patients used global longitudinal RV strain which is an independent predictor of RV failure [19]. Haneya A et al. predicted post LVAD implantation RVF on preoperative Trans Thoracic Echocardiography (TTE) with RV end-diastolic diameter (RVEDD) $>35 \mathrm{~mm}, \mathrm{RVEF}<30 \%$, and right atrial size $>50 \mathrm{~mm}$. Postoperatively on 2 Decho, an increase in RV size, reduced TAPSE suggest worsening Tricuspid valve function [20], also on echocardiography look for IVS position, and pericardial effusion or thrombus over RV in the pericardium as it may compress the right heart causing RVF [15] [21]. 


\section{Management of RVF}

Appropriate patient selection, optimal timing of implant, and preoperative optimization of comorbid conditions are the key aspects that needs attention for successful LVAD placement and minimizing the incidence of RVF. Early identification and management of RVF in the preoperative period and detection of its late manifestation are critical in decreasing mortality and morbidity in patients supported by LVADs. Comparison of various features of RVAD and LVAD is discussed in Table 2 [22].

\subsection{Medical Management}

A pulmonary artery catheter guided management to optimize preoperative increased Pulmonary Vascular Resistance (PVR) and PAP is an ideal approach. A CVP $<15 \mathrm{~mm} \mathrm{Hg}$ ensures decreased RV preload and hepatic and renal congestion [23]. Inodilators such as Dobutamine, Milrinone or Levosimendan which have both inotropic action as well as pulmonary vasodilatation properties \& veno-venous hemodialysis should be considered if CVP $>15 \mathrm{mmHg}$. Inotropes should be weaned off as early as possible as correlation between inotrope duration and mortality has been identified [24]. Besides pharmacological therapy, measures to reduce PVR by adequate mechanical ventilation to reduce hypoxia, hypercarbia, and acidosis are imperative. Preoperative coagulopathy must to be corrected, because it predisposes to bleeding and increases the need for blood

Table 2. Comparison of various features of RVAD vs LVAD [22].

\begin{tabular}{|c|c|c|c|}
\hline Sno & Characteristic & RVAD & LVAD \\
\hline 1 & Principle & $\begin{array}{l}\text { Decompresses the right ventricle and provides blood flow to } \\
\text { pulmonary artery }\end{array}$ & $\begin{array}{l}\text { Decompresses the left ventricle and provides } \\
\text { blood flow to the aorta for systemic circulation }\end{array}$ \\
\hline 2 & Cannulation & $\begin{array}{l}\text { Inflow: Right atrium } \\
\text { Out flow: Main pulmonary artery }\end{array}$ & $\begin{array}{l}\text { Inflow: apex of Left Ventricle (most common site) } \\
\text { Out flow: Ascending Aorta }\end{array}$ \\
\hline 4 & Scope of usage & $\begin{array}{l}\text { Isolated RV failure is less frequently seen, mostly indicated in cases } \\
\text { of RV failure post LVAD implantation and LV failure post } \\
\text { cardiotomy period }\end{array}$ & Isolated LV dysfunction \\
\hline 5 & Indications [22] & $\begin{array}{l}\text { - Mean Right Atrial pressure }>20 \mathrm{mmHg} \\
\text { - Left Atrial pressure }<15 \mathrm{mmHg} \\
\text { - No Tricuspid Regurgitation } \\
\text { - In case of RV dysfunction post LVAD implantation when } \\
\text { unable to maintain LVAD flow }>2.0 \mathrm{lit} / \mathrm{min} / \mathrm{m}^{2} \text { with Right } \\
\text { Atrial pressure }>20 \mathrm{mmHg}\end{array}$ & $\begin{array}{l}\text { - Systolic blood pressure }<90 \mathrm{mmHg} \\
\text { - Left Atrial pressure }>20 \mathrm{mmHg} \\
\text { - Systemic Vascular Resistance }>2100 \\
\text { dynes } / \mathrm{sec} / \mathrm{cm}^{-5}\end{array}$ \\
\hline 6 & Management & $\begin{array}{l}\text { - Flows to maintain systemic output } 2.2 \mathrm{lit} / \mathrm{min} / \mathrm{m}^{2} \text { with Left } \\
\text { Atrial pressure } 15 \mathrm{mmHg} \text { maintaining Right Atrial pressure of } 5 \\
\text { - } 10 \mathrm{~mm} \mathrm{Hg} \\
\text { - activated Partial Thromboplastin Time (aPTT): } 2 \text { - } 2.5 \text { times } \\
\text { normal value, during weaning } 2.5 \text { - } 3 \text { times the normal value } \\
\text { - Activated Clotting Time (ACT): } 180 \text { - } 200 \text { seconds and during } \\
\text { weaning } 250 \text { - } 300 \text { seconds }\end{array}$ & $\begin{array}{l}\text { - Flows to maintain systemic output } 2.2 \\
\text { lit/min } / \mathrm{m}^{2} \text { with Left Atrial pressure in the } \\
\text { range of } 10-15 \mathrm{mmHg} \\
\text { - aPTT: } 2 \text { - } 2.5 \text { times normal value, during } \\
\text { weaning } 2.5 \text { - } 3 \text { times the normal value } \\
\text { - ACT: } 180 \text { - } 200 \text { seconds and during weaning } \\
250 \text { - } 300 \text { seconds }\end{array}$ \\
\hline
\end{tabular}


transfusion causing increased RV preload. Studies on first-generation LVADs have validated the administration of vitamin $\mathrm{K}$ preoperatively or aprotinin intraoperatively to reduce bleeding [25]. Argiriou et al. have used IABP successfully in patients who developed RVF, after IABP placement they found successful reduction of PAP, RVSWI and RAP [9].

\section{Pulmonary Vasodilators}

Inhaled nitric oxide (iNO) is a selective pulmonary vasodilator that reduces PVR. In a study by Potapov et al. iNO was initiated before weaning from cardiopulmonary bypass (CPB) and continued up to 48 hours post-LVAD implantation decreased mean pulmonary artery pressure (mPAP) and increased LVAD flow [26]. However, it did not reduce RVF occurrence, and the maximum benefit was obtained in patients with higher values of mPAP and low pump flow during weaning from CPB [21]. Sildenafil is a phosphodiesterase-5 (PDE-5) inhibitor which lower PVR in persistent PAH has been successfully employed in treatment in post-LVAD implant patients [27].

Milrinone, a phosphodiesterase type 3 inhibitor specific to cardiac and smooth muscle, leads to increased levels of cAMP and, therefore increases cardiac contractility and vasodilation. The beneficial effects come from increased right heart inotropy and decreased PVR [28]. Nesiritide is a recombinant B-type Natriuretic Peptide (BNP) of cardiac origin that causes an increased level of intracellular cGMP, resulting in vasodilatation and natriuresis. It has been shown to produce a dose dependent reduction in PCWP within 15 minutes [29].

\subsection{Surgical Management}

Intraoperative assessment of RVF and degree of Tricuspid Regurgitation (TR) is critical in preventing postoperative RV failure. TR is also aggravated as a result of inter ventricular septum shift caused by LVAD flow. It is common surgical practice to repair Tricuspid Valve with moderate-severe TR and if valve annulus size $>40 \mathrm{~mm}$ at the time of continuous flow left ventricular assist device (CF-LVAD) implantation to decrease the chances of a sudden increase in preload [15] [23]. In cases where right ventricular function compromises pump flow and a patient does not wean off from cardiopulmonary bypass, a temporary right heart bypass can be established. This can be accomplished via pulmonary artery-left atrial graft and has been shown to improve right ventricular function [30].

Intraoperative TEE helps in assessment of RV function after LVAD implantation. Evidence of RVF in TEE with CVP $>20 \mathrm{~mm}$ of $\mathrm{Hg}$ and cardiac index $<2$ $\mathrm{lit} / \mathrm{min} / \mathrm{m}^{2}$ should be considered for RVAD implantation. Finally, pump speed should be set in a range to provide optimal cardiac output, avoiding septal shifts and chamber collapse. This can be achieved intraoperatively, guided by TEE.

\section{Future Research}

It is noted that the need for RVAD implantation is $37 \%$ with the use of pulsatile 
LVADs vs 6\% with CF-LVADs due to better preservation of RV mechanics as a result of lesser LV unloading and interventricular septal shift [2]. The currently available CF-LVADs even though have advantages of smaller size and durability, they are associated with several complications of non-pulsatile circulation such as excessive hemolysis, arteriovenous malformations, gastro intestinal bleeding, pump thrombosis, aortic regurgitation and lower rate of left ventricular recovery [31]. Future research should be focused on developing pulsatility by imparting control algorithms to overcome these adverse effects of continuous flow.

\section{Conclusion}

RVF post LVAD implantation is one of the major causes of postoperative morbidity and mortality. Although there are several scores existing to predict the RVF in the postoperative period, a universally accepted scoring system is still unavailable. There is a need for more sensitive echocardiography parameters to detect the RVF before its onset with clinical manifestations. The role of using pulmonary vasodilators like inhaled $\mathrm{NO}$ and sildenafil in improving RV function is still debatable. Surgery should be performed in a meticulous manner to avoid postoperative complications that may worsen the RV function.

\section{Funding}

No funding source.

\section{Ethical Approval}

Not applicable.

\section{Human Rights}

Not applicable.

\section{Conflicts of Interest}

No conflict of interests.

\section{References}

[1] Kirklin, J.K., Naftel, D.C., Kormos, R.L., Stevenson, L.W., Pagani, F.D., Miller, M.A., Baldwin, J.T. and Young, J.B. (2013) Fifth INTERMACS Annual Report: Risk Factor Analysis from More than 6,000 Mechanical Circulatory Support Patients. The Journal of Heart and Lung Transplantation, 32, 141-156. https://doi.org/10.1016/j.healun.2012.12.004

[2] Kormos, R.L., Teuteberg, J.J., Pagani, F.D., Russell, S.D., John, R., Miller, L.W., et al. (2010) Right Ventricular Failure in Patients with the HeartMate II Continuous-Flow Left Ventricular Assist Device: Incidence, Risk Factors, and Effect on Outcomes. The Journal of Thoracic and Cardiovascular Surgery, 139, 1316-1324. https://doi.org/10.1016/j.jtcvs.2009.11.020

[3] Matthews, J.C., Koelling, T.M., Pagani, F.D. and Aaronson, K.D. (2008) The Right Ventricular Failure Risk Score a Pre-Operative Tool for Assessing the Risk of Right 
Ventricular Failure in Left Ventricular Assist Device Candidates. Journal of the American College of Cardiology, 51, 2163-2172. https://doi.org/10.1016/j.jacc.2008.03.009

[4] Kukucka, M., Stepanenko, A., Potapov, E., Krabatsch, T., Redlin, M., Mladenow, A., et al. (2011) Right-to-Left Ventricular End-Diastolic Diameter Ratio and Prediction of Right Ventricular Failure with Continuous-Flow Left Ventricular Assist Devices. The Journal of Heart and Lung Transplantation, 30, 64-69. https://doi.org/10.1016/j.healun.2010.09.006

[5] Fida, N., Loebe, M., Estep, J.D. and Guha, A. (2015) Predictors and Management of Right Heart Failure after Left Ventricular Assist Device Implantation. Methodist Debakey Cardiovascular Journal, 11, 18-23.

[6] Topilsky, Y., Hasin, T., Oh, J.K., Borgeson, D.D., Boilson, B.A., Schirger, J.A., et al. (2011) Echocardiographic Variables after Left Ventricular Assist Device Implantation Associated with Adverse Outcome. Circulation: Cardiovascular Imaging, 4, 648-661. https://doi.org/10.1161/CIRCIMAGING.111.965335

[7] Farrar, D.J., Compton, P.G., Hershon, J.J., Fonger, J.D. and Hill, J.D. (1985) Right Heart Interaction with the Mechanically Assisted Left Heart. World Journal of Surgery, 9, 89-102. https://doi.org/10.1007/BF01656260

[8] Farrar, D.J. (1994) Ventricular Interactions during Mechanical Circulatory Support. Seminars in Thoracic and Cardiovascular Surgery, 6, 163-168.

[9] Argiriou, M., Kolokotron, S.M., Sakellaridis, T., Argiriou, O., Charitos, C., Zarogoulidis, P., Katsikogiannis, N., Kougioumtzi, I., Machairiotis, N., Tsiouda, T., Tsakiridis, K. and Zarogoulidis, K. (2014) Right Heart Failure Post Left Ventricular Assist Device Implantation. Journal of Thoracic Disease, 6, S52-S59.

[10] Fitzpatrick, J.R. III, Frederick, J.R., Hsu, V.M., Kozin, E.D., Lou O’Hara, M., Howell, E., et al. (2008) Risk Score Derived from Pre-Operative Data Analysis Predicts the Need for Biventricular Mechanical Circulatory Support. The Journal of Heart and Lung Transplantation, 27, 1286-1292.

https://doi.org/10.1016/j.healun.2008.09.006

[11] Drakos, S.G., Janicki, L., Horne, B.D., Kfoury, A.G., Reid, B.B., Clayson, S., et al. (2010) Risk Factors Predictive of Right Ventricular Failure after Left Ventricular Assist Device Implantation. American Journal of Cardiology, 105, 1030-1035. https://doi.org/10.1016/j.amjcard.2009.11.026

[12] Ochiai, Y., McCarthy, P.M., Smedira, N.G., Banbury, M.K., Navia, J.L., Feng, J.Y., et al. (2002) Predictors of Severe Right Ventricular Failure after Implantable Left Ventricular Assist Device Insertion: Analysis of 245 Patients. Circulation, 106, I198-202.

[13] Pagani, F.D., Miller, L.W., Russell, S.D., Aaronson, K.D., John, R., Boyle, A.J., et al. (2009) Extended Mechanical Circulatory Support with a Continuous-Flow Rotary Left Ventricular Assist Device. Journal of the American College of Cardiology, 54, 312-321. https://doi.org/10.1016/j.jacc.2009.03.055

[14] Farrar, D.J., Hill, J.D., Pennington, D.G., McBride, L.R., Holman, W.L., Kormos, R.L., et al. (1997) Preoperative and Postoperative Comparison of Patients with Univentricular and Biventricular Support with the Thoratec Ventricular Assist Device as a Bridge to Cardiac Transplantation. The Journal of Thoracic and Cardiovascular Surgery, 113, 202-209. https://doi.org/10.1016/S0022-5223(97)70416-1

[15] Meineri, M., Van Rensburg, A.E. and Vegas, A. (2012) Right Ventricular Failure after LVAD Implantation: Prevention and Treatment. Best Practice \& Research Clinical Anaesthesiology, 26, 217-229. https://doi.org/10.1016/j.bpa.2012.03.006

[16] Verhaert, D., Mullens, W., Borowski, A., Popovic, Z.B., Curtin, R.J., Thomas, J.D., 
et al. (2010) Right Ventricular Response to Intensive Medical Therapy in Advanced Decompensated Heart Failure. Circulation: Heart Failure, 3, 340-346. https://doi.org/10.1161/CIRCHEARTFAILURE.109.900134

[17] Kato, T.S., Farr, M., Schulze, P.C., Maurer, M., Shahzad, K., Iwata, S., et al. (2012) Usefulness of Two-Dimensional Echocardiographic Parameters of the Left Side of the Heart to Predict Right Ventricular Failure after Left Ventricular Assist Device Implantation. American Journal of Cardiology, 109, 246-251.

https://doi.org/10.1016/j.amjcard.2011.08.040

[18] Aissaoui, N., Paluszkiewicz, L., Martin Gorria, G., et al. (2013) Assessment of Right Ventricle before the Implantation of Left Ventricular Assist Device by Echocardiographic Parameters (ARVADE). The Journal of Heart and Lung Transplantation, 32, S234-S235. https://doi.org/10.1016/j.healun.2013.01.599

[19] Grant, A.D., Smedira, N.G., Starling, R.C. and Marwick, T.H. (2012) Independent and Incremental Role of Quantitative Right Ventricular Evaluation for the Prediction of Right Ventricular Failure after Left Ventricular Assist Device Implantation. Journal of the American College of Cardiology, 60, 521-528. https://doi.org/10.1016/j.jacc.2012.02.073

[20] Haneya, A., Philipp, A., Puehler, T., Rupprecht, L., Kobuch, R., Hilker, M., et al. (2012) Temporary Percutaneous Right Ventricular Support Using a Centrifugal Pump in Patients with Postoperative Acute Refractory Right Ventricular Failure after Left Ventricular Assist Device Implantation. European Journal of Cardio-Thoracic Surgery, 41, 219-223. https://doi.org/10.1016/j.ejcts.2011.04.029

[21] Kukucka, M., Potapov, E., Stepanenko, A., Weller, K., Mladenow, A., Kuppe, H. and Habazettl, H. (2011) Acute Impact of Left Ventricular Unloading by Left Ventricular Assist Device on the Right Ventricle Geometry and Function: Effect of Nitric Oxide Inhalation. The Journal of Thoracic and Cardiovascular Surgery, 141, 1009-1014. https://doi.org/10.1016/j.jtcvs.2010.08.010

[22] Bojar, R.M. (2011) Cardiovascular Management. In: Bojar, R.M., Ed., Manual of Perioperative Care in Adult Cardiac Surgery, John Wiley \& Sons, Ltd., Hoboken, 479. Table 11.7.

[23] Slaughter, M.S., Pagani, F.D., Rogers, J.G., Miller, L.W., Sun, B., Russell, S.D., et al. (2010) Clinical Management of Continuous-Flow Left Ventricular Assist Devices in Advanced Heart Failure. The Journal of Heart and Lung Transplantation, 29, S1-S39. https://doi.org/10.1016/j.healun.2010.01.011

[24] Schenk, S., McCarthy, P.M., Blackstone, E.H., Feng, J.Y., Starling, R.C., Navia, J.L., et al. (2006) Duration of Inotropic Support after Left Ventricular Assist Device Implantation: Risk Factors and Impact on Outcome. The Journal of Thoracic and Cardiovascular Surgery, 131, 447-454.https://doi.org/10.1016/j.jtcvs.2005.09.031

[25] Kaplon, R.J., Gillinov, A.M., Smedira, N.G., Kottke-Marchant, K., Wang, I.-W., Goormastic, M. and McCarthy, P.M. (1999) Vitamin K Reduces Bleeding in Left Ventricular Assist Device Recipients. The Journal of Heart and Lung Transplantation, 18, 346-350. https://doi.org/10.1016/S1053-2498(98)00066-7

[26] Potapov, E., Meyer, D., Swaminathan, M., Ramsay, M., El Banayosy, A., Diehl, C., et al. (2011) Inhaled Nitric Oxide after Left Ventricular Assist Device Implantation: A Prospective, Randomized, Double-Blind, Multicenter, Placebo-Controlled Trial. The Journal of Heart and Lung Transplantation, 30, 870-878. https://doi.org/10.1016/j.healun.2011.03.005

[27] Tedford, R.J., Hemnes, A.R., Russell, S.D., Wittstein, I.S., Mahmud, M., Zaiman, A.L., et al. (2008) PDE5A Inhibitor Treatment of Persistent Pulmonary Hypertension after Mechanical Circulatory Support. Circulation: Heart Failure, 1, 213-219. 
https://doi.org/10.1161/CIRCHEARTFAILURE.108.796789

[28] Kihara, S., Kawai, A., Fukuda, T., Yamamoto, N., Aomi, S., Nishida, H., et al. (2002) Effects of Milrinone for Right Ventricular Failure after Left Ventricular Assist Device Implantation. Heart \& Vessels, 16, 69-71.

https://doi.org/10.1007/s380-002-8320-Z

[29] Abraham, W.T., Cheng, M.L., Smoluk, G., et al. (2005) Clinical and Hemodynamic Effects of Nesiritide (Btype Natriuretic Peptide) in Patients with Decompensated Heart Failure Receiving Beta Blockers. Congestive Heart Failure, 11, 59-64.

[30] Spillner, J., Amerini, A., Hatam, N., Rex, S., Pott, F., Goetzenich, A., et al. (2011) Pulmono-Atrial Shunt and Lung Assist to Treat Right Ventricular Failure. Frontiers in Bioscience, 16, 2342-2351. https://doi.org/10.2741/3857

[31] Cheng, A., Williamitis, C.A. and Slaughter, M.S. (2014) Comparison of Continuous-Flow and Pulsatile-Flow Left Ventricular Assist Devices: Is There an Advantage to Pulsatility? Annals of Cardiothoracic Surgery, 3, 573-581.

https://doi.org/10.3978/j.issn.2225-319x.2014.08.24

\section{List of Abbreviations}

$\begin{array}{ll}\text { LVAD } & \text { Left Ventricular Assist Device } \\ \text { INTERMACS } & \begin{array}{l}\text { Interagency Registry for Mechanically Assisted Circulatory } \\ \text { Support }\end{array} \\ \text { BTT } & \text { Bridge To Transplant } \\ \text { DT } & \text { Destination Therapy } \\ \text { TR } & \text { Tricuspid Regurgitation } \\ \text { RVF } & \text { Right Ventricular Failure } \\ \text { TEE } & \text { Trans Esophageal Echocardiography } \\ \text { RVSWI } & \text { Right Ventricular Stroke Work Index } \\ \text { IABP } & \text { Intra Aortic Balloon Pump } \\ \text { BUN } & \text { Blood Urea Nitrogen } \\ \text { RVAD } & \text { Right Ventricular Assist Device } \\ \text { CVP } & \text { Central Venous Pressure } \\ \text { PCWP } & \text { Pulmonary Capillary Wedge Pressure } \\ \text { PVR } & \text { Pulmonary Vascular Resistance } \\ \text { BiVAD } & \text { Bi Ventricular Assist Device } \\ \text { PAP } & \text { Pulmonary Artery Pressure } \\ \text { ROC curve } & \text { Receiver Operator Characteristic curve } \\ \text { RVEDD } & \text { Right Ventricular End Diastolic Dimension } \\ \text { iNO } & \text { Inhaled Nitric Oxide } \\ \text { PDE-5 } & \text { Phospho Di Esterase-5 } \\ \text { BNP } & \text { B-type Natriuretic Peptide } \\ \text { CF-LVAD } & \text { Continuous Flow Left Ventricular Assist Device } \\ \text { aPTT } & \text { activated Partial Thromboplastin Time } \\ \text { ACT } & \text { Activated Clotting Time } \\ \end{array}$

